\title{
EFFECT OF AN IN-FLIGHT VERTICAL ACCELEROMETER CALIBRATION ON LANDING ACCURACY AFTER BARO-INERTIAL SYSTEM FAILURE
}

\author{
Man NGUYEN (1)*, Vyacheslav KOSTIUKOV (D), Cap TRAN (10) \\ Department of Automated Complexes of Orientation and Navigation Systems, \\ Moscow Aviation Institute, Moscow, Russia
}

Received 26 March 2020; accepted 01 April 2020

\begin{abstract}
An issue of improving flight safety during landing with an inertial navigation system (INS) and a failed barometric altimeter is considered. In this paper, we propose a specific algorithm for in-flight calibration of the vertical channel of INS. Accordingly, the baro-inertial integration algorithm using a discrete five-state Kalman filter will be performed during a particular flight maneuver before landing. As a result, it is possible to estimate not only the bias of vertical accelerometer but also its scale factor, which is too small to be defined by a usual in-flight calibration algorithm. After applying the proposed algorithm, the flight management system can provide a safe landing with a standalone INS. The algorithm's performance is assessed by simulating complete mathematical models of aircraft motion and control systems. The impact of calibrated bias and scale factor of vertical accelerometer on the altitude estimation error is provided through an analysis.
\end{abstract}

Keywords: accelerometer, barometric altimeter, calibration maneuver, baro-inertial failure, Kalman filter, automatic landing.

\section{Introduction}

Inertial navigation system (INS) is one of the most effective devices that provide an estimation of aircraft state to ensure the accuracy and safety of its motion. In contrast to other navigation and guidance systems, INS is completely autonomous and free of external influences such as weather and electromagnetic interferences. However, the major disadvantage of an INS is the unlimited growth of its errors over time, especially in the vertical channel (Babich, 1991; O'Donnel, 1964), making the long-term application of standalone INS inefficient. In order to overcome this drawback, integration of INS and other position and velocity measurements such as Global Navigation Satellite System (GNSS), radio systems, or barometric altimeter (BA) (Schmidt, 2010; Siouris, 1993) is used. By using signals from such systems, it is possible to obtain more accurate estimates of aircraft state even when the characteristics of INS are poorly known. Nevertheless, the advantage of INS and noninertial navigation sensors integration does not eliminate the need for in-flight inertial sensors calibration, since it allows us to reduce position errors when only a standalone INS is available due to external measurement failure.
As is well known, GNSS is the most common aiding system that can give an absolute drift-free position estimate with high accuracy (Groves, 2013; Mohinder et al., 2007). However, due to the effect of satellite geometry and aircraft maneuver, GNSS provides less accurate altitude estimation (Farrel \& Barth, 1998; Kim \& Sukkarieh, 2003). Hence, an additional aiding system, such as BA, is needed to improve the vertical channel (Ausman, 1991; Babich, 1991; Farrell \& Barth, 1998; Kim \& Sukkarieh, 2003; O’Donnel, 1964; Sobolev, 1994). Many researchers have developed various algorithms for baro-inertial integration, using simple vertical channel damping-loop mechanizations or complex forms of Kalman filter. The results show that using BA additionally to integrated navigation systems allows us to obtain a more reliable and accurate navigation solution. Furthermore, it is shown that baro-inertial integration meets the FAA requirement for a Category I precision approach (Gray \& Maybeck, 1995).

However, in most publications, the authors used incomplete mathematical models of integrated systems. For example, the dynamic error of BA, i.e., lag in static pressure transmission, is not taken into account (Bevermeier et al., 2010; Kim \& Sukkarieh, 2003; Sokolovi et al.,

${ }^{*}$ Corresponding author. E-mail: nguennm@mai.ru 
2014), or the accelerometer error model including only bias is considered (Babich, 1991; Sobolev, 1994). In some cases, additional information from a non-standard atmosphere condition (Jafar et al., 2018) or map-matching with a topographic map (Bevermeier et al., 2010) is included for compensating the BA bias caused by deviation of sea-level temperature and static pressure from their nominal values used in BA computation. This leads to the use of a more complex structure of integration algorithm, which can be avoided by a suitable selection of measurement information from BA output, as done in this work. Besides that, the main issue of these investigations is to provide a more stable and accurate altitude estimation with BA aiding. For this purpose, it is sufficient to define only the accelerometer bias since the rest of its characteristics are quite small (for aviation-grade INS). As a result, in the event of BA failure, the altitude estimation is not exact enough to ensure the safety of aircraft motion, especially in such complicated and dangerous phase of flight as landing, as shown in simulation results of this paper.

It is very important to have accurate altitude information during landing because any hazardous situation could occur if either the pilot or automatic control system receives erroneous altitude estimates. Numerous aviation disasters have been caused by the air data computer failure (Jeb, 2019; Luiz, 2013). Among them were the accidents involving Air India Flight 855 (January 1978), Birgenair Flight 301 (February 1996), Adam Air Flight 574 (January 2007), Turkish Airlines Flight 1951 (February 2009), Air France Flight 447 (June 2009), United Airlines Flight N41140 (October 2013), or the recent Boeing 737 Max crashes. The primary cause of these disasters is blockage of the Pitot-static systems (AAIU, 2016; BEA, 2012; KNKT, 2018), among which the BA failure due to the static port blockage by water or airframe icing is more dangerous (FAA, 2016).

Consequently, improving flight safety in the event of BA failure is always highly relevant. In this paper, we propose an algorithm that provides calibration of the vertical accelerometer of INS during a specific flight maneuver before landing. This method allows us to estimate not only the accelerometer bias but also its scale factor, which is too small (aviation-grade INS) to be defined by a "normal" inflight calibration (i.e., without any calibration maneuver). Using the calibrated bias and scale factor for additional compensation of INS error, the flight management system can provide a safe landing when the BA is failed.

Fully observable information of the measurement model including vertical accelerometer and BA is derived for calibration using a discrete Kalman filter, one of the most effective and widely used methods for realizing baro-inertial integration. The effectiveness of the proposed calibration algorithm follows an analysis of mathematical simulation results. The reliability of the obtained results is based on the fact that the investigation in this paper is performed using complete models of related dynamic systems (aircraft and landing control loops). In addition, an example of analyzing the impact of the calibrated bias and scale factor of a vertical accelerometer on altitude estimation error during landing is given.

\section{Mathematical model of Kalman filter calibrating the vertical accelerometer}

To describe the vertical channel of INS, we consider that the residual error (after all compensations) in the estimate of vertical acceleration, measured by the vertical accelerometer, is caused by the following error sources:

1) an inaccuracy of setting the bias $\Delta a_{0}$;

2) an inaccuracy of setting the scale factor $\delta k_{a}$;

3 ) measurement noise $\xi$ with zero mean and standard deviation $\sigma_{a}$.

Respectively, a mathematical model for the vertical accelerometer of INS expresses the output $a_{m}$ as:

$$
a_{m}=\left(1+\delta k_{a}\right) a_{y}+\Delta a_{0}+\xi,
$$

where $a_{y}$ - vertical acceleration of an aircraft.

Hence, the vertical acceleration can be estimated by the following equation:

$$
a_{y}=\frac{a_{m}-\Delta a_{0}-\xi}{1+\delta k_{a}} \approx\left(a_{m}-\Delta a_{0}-\xi\right)\left(1-\delta k_{a}\right),
$$

since $\delta k_{a} \ll 1$.

The model of BA output is adopted as (Babich, 1991):

$$
\frac{d}{d t} h_{m}=\frac{1}{\tau}\left(-h_{m}+h+\Delta h_{0}+\mu\right),
$$

where $h_{m}$ - output signal; $h$ - aircraft altitude; $\Delta h_{0}$ - bias; $\tau$ - time constant, defined by (4); $\mu$ - measurement noise with zero mean and standard deviation $\sigma_{b}$.

The time constant $\tau$ of BA depends on the static pressure and temperature of the atmosphere (Lawford \& Nippress, 1983; Sobolev, 1994):

$$
\tau=\tau_{0} \frac{P_{0}}{P_{h}} \sqrt{\left(\frac{T_{h}}{T_{0}}\right)^{3}} \frac{T_{0}+110.4}{T_{h}+110.4},
$$

where $\tau_{0}$ - time constant at sea-level; $P_{0}, T_{0}$ - sea-level static pressure and temperature of the atmosphere; $P_{h}, T_{h}-$ static pressure and temperature of the atmosphere at $h$.

From (3), the discrete model of BA output can be represented in the following form (Sobolev, 1994):

$$
\begin{aligned}
& h_{m}(k+1)=e^{-\Delta T / \tau} h_{m}(k)+\left(1-e^{-\Delta T / \tau}\right) h(k)+ \\
& \left(1-e^{-\Delta T / \tau}\right) \Delta h_{0}+\left(1-e^{-\Delta T / \tau}\right) \mu(k),
\end{aligned}
$$

here $\Delta T$ - sample time.

Essentially, the algorithm calibrating a vertical accelerometer by integrating its output and BA output is based on the comparison of the flight altitude changes estimated by these sensors separately. Since BA measures an absolute value of aircraft altitude, it is necessary to remove the bias $\Delta h_{0}$ from the output $h_{m}$ (5) to estimate the altitude change. For this purpose, the difference $\Delta h_{m}=h_{m}-h_{m_{0}}$ between current BA output and the initial one is used as a measurement for the Kalman filter: 


$$
\begin{aligned}
& \Delta h_{m}(k+1)=h_{m}(k+1)-h_{m}(0)= \\
& e^{-\Delta T / \tau} \Delta h_{m}(k)+\left(1-e^{-\Delta T / \tau}\right) \Delta h(k)+\left(1-e^{-\Delta T / \tau}\right) \eta(k), \text { (6) }
\end{aligned}
$$

where $\Delta h(k)=h(k)-h_{0}$ - altitude change since the initial moment; $h_{0}$ - altitude at the initial moment; $\eta=\mu-\mu_{0}$, $\mu_{0}$ - a sample of BA measurement noise at the initial moment.

The Kalman filter state vector is established as:

$$
\{x\}=\left[\Delta h, V_{y}, \Delta a_{0}, \delta k_{a}, \Delta h_{b}\right]^{T},
$$

where $V_{y}$ - vertical speed; $\Delta h_{b}$ - predicted variation of BA output since initial moment (without consideration of measurement noise).

Thus, the evolution of Kalman filter state is described by the following system of equations:

$$
\begin{aligned}
& \Delta h(k+1)=\Delta h(k)+\Delta T V_{y}(k)+\frac{1}{2} \Delta T^{2} a_{y}(k) ; \\
& V_{y}(k+1)=V_{y}(k)+\Delta T a_{y}(k) ; \\
& \Delta a_{0}(k+1)=\Delta a_{0}(k) ; \\
& \delta k_{a}(k+1)=\delta k_{a}(k) ; \\
& \Delta h_{b}(k+1)=\left(1-e^{-\Delta T / \tau}\right) \Delta h(k)+e^{-\Delta T / \bar{\tau}} \Delta h_{b}(k),
\end{aligned}
$$

with $\bar{\tau}$ - estimation of BA time constant.
Taking into account (2) and (7-12) we have:

$$
x(k+1)=\mathbf{A} x(k)+\mathbf{B} u(k)+\mathbf{G}_{p} \xi(k),
$$

here

$\mathbf{A}=\left[\begin{array}{ccccc}1 & \Delta T & -\frac{1}{2} \Delta T^{2}\left(1-\delta k_{a}\right) & -\frac{1}{2} \Delta T^{2} a_{m} & 1-e^{-\Delta T / \bar{\tau}} \\ 0 & 1 & -\Delta T\left(1-\delta k_{a}\right) & \Delta T a_{m} & 0 \\ 0 & 0 & 1 & 0 & 0 \\ 0 & 0 & 0 & 1 & 0 \\ 1-e^{-\Delta T / \bar{\tau}} & 0 & 0 & e^{-\Delta T / \bar{\tau}} & e^{-\Delta T / \bar{\tau}}\end{array}\right]$, $\mathbf{B}=\left[\begin{array}{c}\frac{1}{2} \Delta T^{2} \\ \Delta T \\ 0 \\ 0 \\ 0\end{array}\right], \quad \mathbf{G}_{p}=\left[\begin{array}{c}-\frac{1}{2} \Delta T^{2}\left(1-\delta k_{a}\right) \\ -\Delta T\left(1-\delta k_{a}\right) \\ 0 \\ 0 \\ 0\end{array}\right], \quad\{u\}=\left[a_{m}\right]$

The measurement for Kalman filter can be formed as follows, according to (6) and (7):

$$
y(k+1)=\mathbf{C} x(k)+\mathbf{G}_{m} \eta(k),
$$

where $\{y\}=\left[\Delta h_{m}\right], \mathbf{C}=[0,0,0,0,1]^{T}, \mathbf{G}_{m}=\left[1-e^{-\Delta T / \bar{\tau}}\right]$.

The block diagram of the Kalman filter using (13) and (14) in a recursive loop is shown in Figure 1.

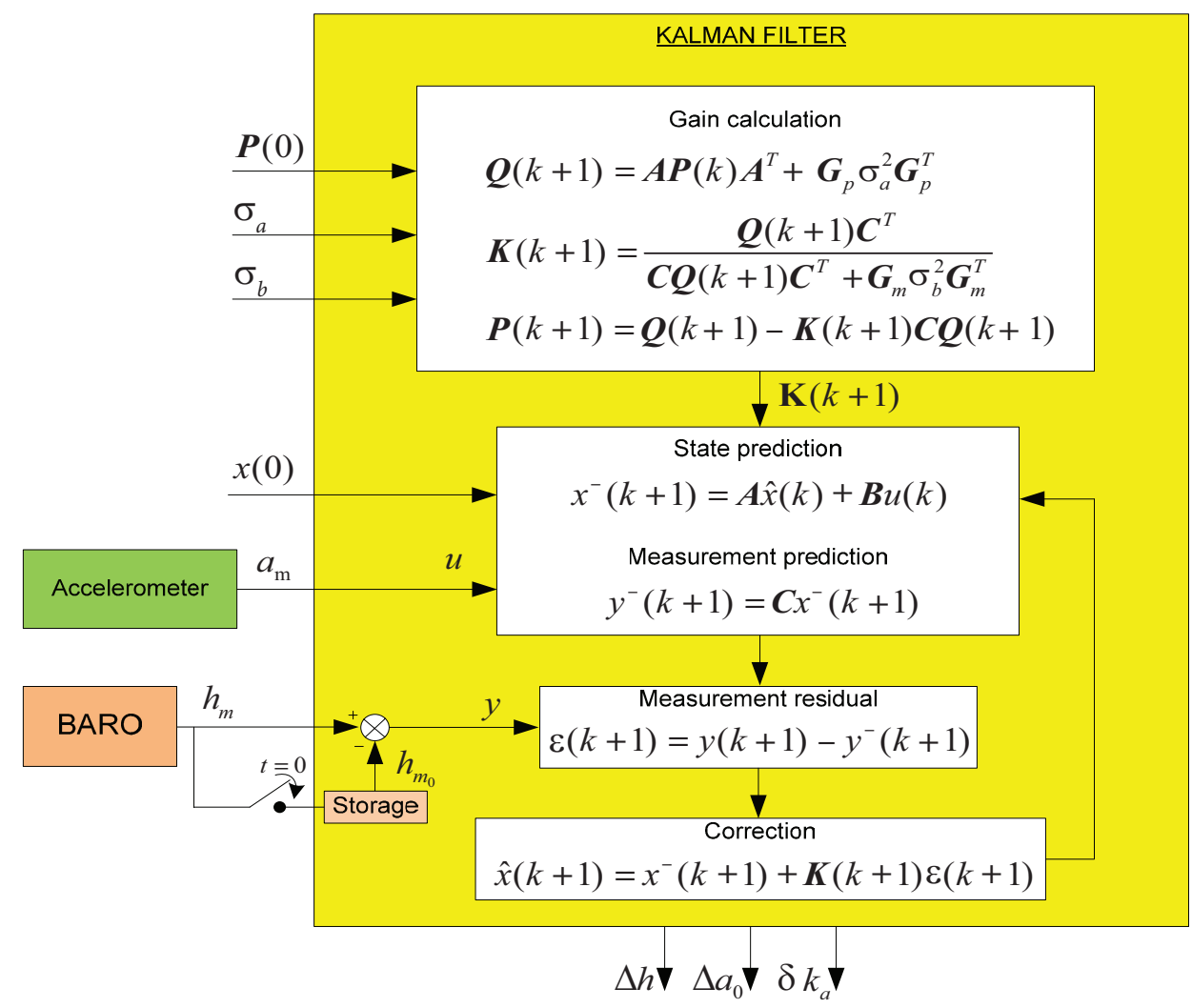

Figure 1. Calibration Kalman filter 


\section{Mathematical model of an aircraft}

A nonlinear mathematical model of longitudinal motion of a passenger aircraft is used for modeling the flight during landing. Due to the limited flight time, the aircraft motion model with a flat earth is considered. Thus, the flight dynamics equations are as follow (Zaporozhets \& Kostiukov, 1992):

$$
\begin{aligned}
& \frac{d}{d t} V=\frac{T \cos \alpha-X}{m}-g \sin \theta \\
& \frac{d}{d t} \theta=\frac{T \sin \alpha+Y-m g \cos \theta}{m V} \\
& \frac{d}{d t} \omega_{z}=\frac{M_{z}}{I_{z}} \\
& \frac{d}{d t} \vartheta=\omega_{z} ; \\
& \frac{d}{d t} \alpha=\omega_{z}-\frac{d}{d t} \theta \\
& \frac{d}{d t} h=V \sin \theta
\end{aligned}
$$

where $T$ - thrust; $X, Y$ - drag and lift forces; $M_{z}$ - pitching moment; $V$ - speed; $\alpha$ - angle of attack; $\theta$ - flight path angle; $\omega_{z}$ - rate of pitch angle; $\vartheta$ - pitch angle; $h$ - altitude; $m$ - mass; $I_{z}$ - inertial moment.

Figure 2 provides a definition of all forces and moments acting on an aircraft, as well as angles indicated in the flight dynamics equations (15-20).

The aerodynamic model is determined by formulas (Zaporozhets \& Kostiukov, 1992):

$$
C_{y}=C_{y}^{\alpha} \alpha+C_{y}^{\delta_{e}} \delta_{e}+C_{y}^{\phi} \phi_{s}
$$

$$
\begin{gathered}
C_{x}=C_{x 0}+A C_{y}+B C_{y}^{2}+\left(C_{x}^{\delta_{e}}+C_{x}^{\delta_{e} \alpha} \alpha+C_{x}^{\delta_{e} \alpha^{2}} \alpha^{2}\right) \delta_{\mathrm{e}}+C_{x}^{\phi} \phi_{s} ; \\
m_{z}=m_{z 0}+m_{z}^{\alpha} \alpha+m_{z}^{\alpha^{2}} a^{2}+m_{z}^{\delta_{e}} \delta_{e}+m_{z}^{\phi} \phi_{s}+ \\
\left(m_{z}^{\omega_{z}} \omega_{z}+m_{z}^{\alpha} \frac{d}{d t} \alpha\right) b / V+\frac{C_{y}\left(x_{T}-25\right)}{100},
\end{gathered}
$$

where $C_{x}-$ drag coefficient; $C_{y}-$ lift coefficient; $m_{2}-$ pitch moment coefficient; $\delta_{\mathrm{e}}$ - elevator deflection; $\phi_{s}$ - angle of horizontal stabilizer; $b$ - wingspan; $x_{T}$ - center of gravity.

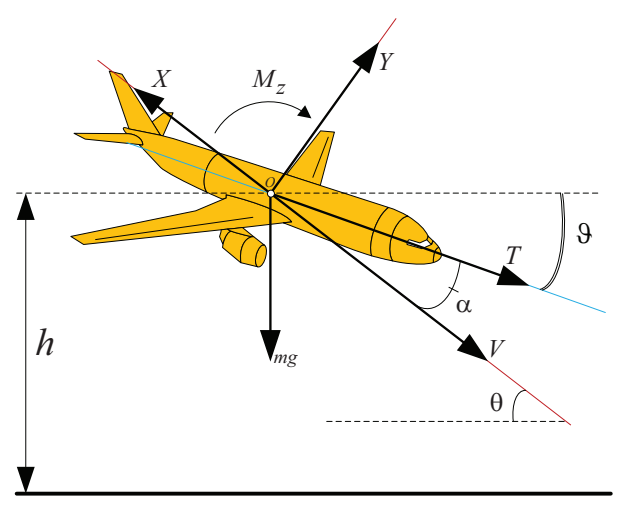

Figure 2. Forces and moments acting on an aircraft
In the landing configuration (Zaporozhets \& Kostiukov, 1992): $\phi_{s}=3 \mathrm{deg}, x_{T}=25 \%$ m.a.c., $C_{y}^{\alpha}=0.093$, $C_{y}^{\delta_{e}}=0.006, C_{y}^{\phi}=0.0145, C_{x 0}=0.0586, A=-0.0518$, $B=0.0876, C_{x}^{\delta_{e}}=-1.920 \mathrm{E}-4, C_{x}^{\delta_{e} \alpha}=8.11 \mathrm{E}-5, C_{x}^{\delta_{e} \alpha^{2}}=0.0$, $C_{x}^{\phi}=-2.33 \mathrm{E}-3, m_{z 0}=0.0515, m_{z}^{\alpha}=-3.215 \mathrm{E}-2, m_{z}^{\alpha^{2}}=$ $5.3 \mathrm{E}-4, m_{z}^{\delta_{e}}=-0.0185, m_{z}^{\phi}=-0.0465, m_{z}^{\omega_{z}}=-12.9, m_{z}^{\alpha^{\prime}}=$ -5.0 .

\section{Calibration maneuver planning}

In order to design a maneuver for in-flight accelerometer calibration let's rewrite equation (2) for vertical acceleration estimation:

$$
a_{y} \approx\left(a_{m}-\Delta a_{0}\right)\left(1-\delta k_{a}\right) \approx a_{m}\left(1-\delta k_{a}\right)-\Delta a_{0},
$$

where $\Delta a_{0} \delta k_{a}$ is omitted because it is very small in comparison with $\Delta a_{0}$.

The essence of an algorithm calibrating the accelerometer with output structure (1) is to find such values of $\Delta a_{0}$ and $\delta k_{a}$, which maximally reduce the difference between altitude estimate obtained by double integration of vertical acceleration $a_{y}$ estimated by (24) and the one received directly from BA output. According to (24), the bias $\Delta a_{0}$ can be well defined in a normal flight condition without any vertical acceleration, i.e., when $a_{m} \approx 0$, and vice versa, the scale factor $\delta k_{a}$ can be calibrated only under a significant vertical maneuver. Therefore, in this paper, to study the effect of a flight maneuver on the accuracy of vertical accelerometer calibration, we suggest changing the aircraft trajectory with the command illustrated in Figure $3\left(h_{0}-\right.$ initial altitude, $h_{d}$ - desired value of altitude, $\Delta h_{r}$ - reference altitude change, $\Delta T_{c m d}-$ command duration, $t_{0}-$ starting time of maneuver).

To perform such maneuver as given in Figure 3, an altitude/speed hold autopilot is used (see Figure 4 for detail).

Respectively, the control signals are:

$$
\begin{aligned}
& u_{T}=u_{T 0}+k_{V}\left(V_{d}-\hat{V}\right)+k_{a} \hat{a}+k_{h V}\left(\Delta h_{r}-\hat{\Delta} h\right) \\
& u_{e}=u_{e 0}+k_{\omega} \hat{\omega}_{z}+k_{\theta} \hat{\theta}+k_{V h}\left(V_{d}-\hat{V}\right)+k_{h}\left(\Delta h_{r}-\hat{\Delta} h\right)
\end{aligned}
$$

where $u_{T 0}, u_{e 0}$ - program controls corresponding to the trim condition (level flight at $500 \mathrm{~m}$ with speed $80 \mathrm{~m} / \mathrm{s}$ ); $V_{d}$ - desired speed; $\Delta h_{r}$ - reference altitude change; $\hat{V}$, $\hat{a}, \hat{\omega}_{z}, \hat{\theta}, \hat{\Delta} h$ - estimated value of speed, acceleration,

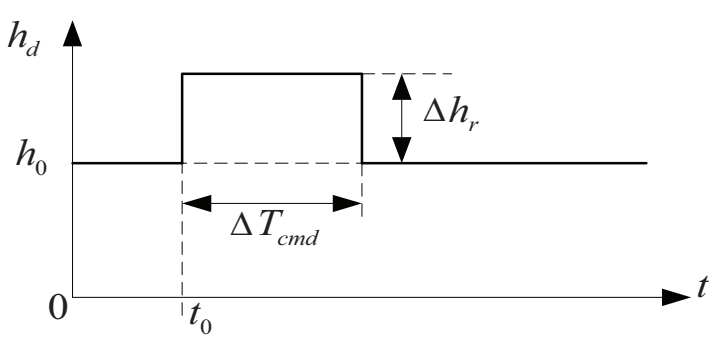

Figure 3. Altitude change command 


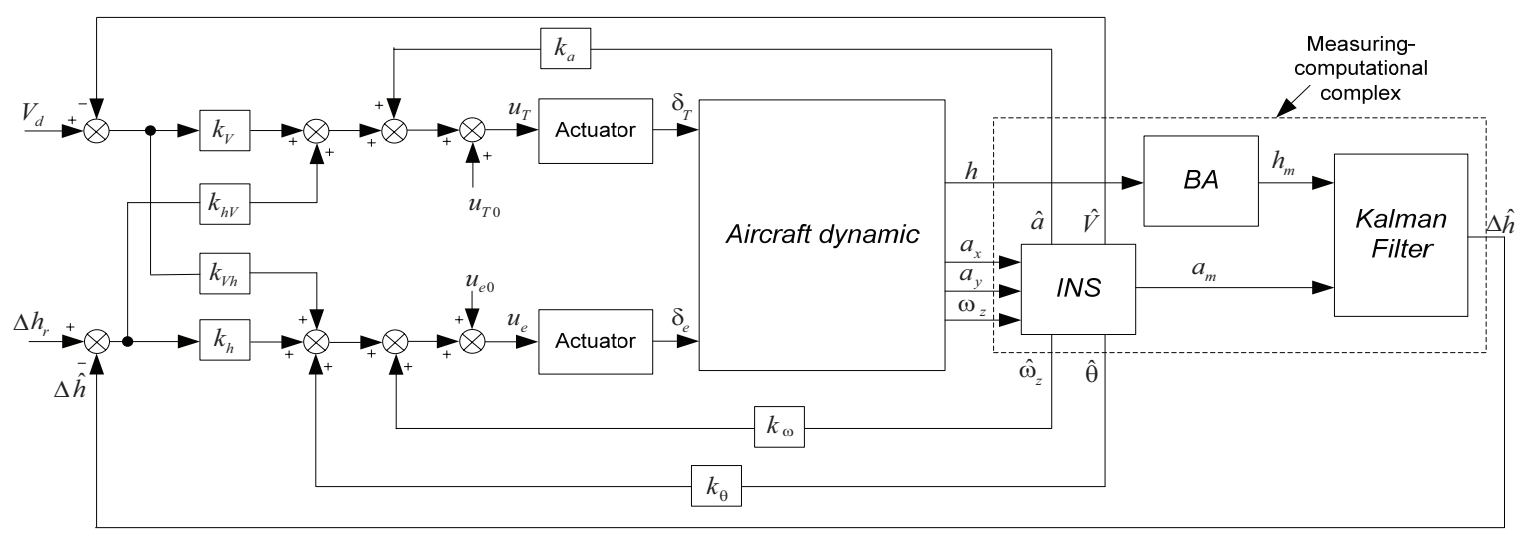

Figure 4. An altitude/speed hold autopilot

pitch angle rate, flight path angle and altitude change; $k_{V}=0.08, k_{a}=-0.23, k_{h V}=-0.018, k_{\omega}=0.41, k_{\theta}=0.715$, $k_{h V}=0.002, k_{h}=-0.07$.

The dynamic of throttle and elevator actuators is (Brian \& Frank, 2003):

$$
\begin{gathered}
\frac{d}{d t} \delta_{T}=\frac{u_{T}-\delta_{T}}{T_{T}} \\
\frac{d}{d t} \delta_{e}=\frac{u_{e}-\delta_{e}}{T_{e}}
\end{gathered}
$$

where $\delta_{T}, \delta_{e}-$ throttle and elevator deflection; $T_{T}=5 \mathrm{~s}$, $T_{e}=0.1 \mathrm{~s}-$ time constant of actuators.

\section{Analysis of results}

To assess the feasibility of the proposed in-flight calibration algorithm, a simulation was performed, using the aviation-grade accelerometer (Groves, 2015) with $\Delta a_{0}=$ $0.001 \mathrm{~m} / \mathrm{s}^{2}, \delta k_{a}=1000 \mathrm{ppm}, \sigma_{a}=0.0062 \mathrm{~m} / \mathrm{s}^{2}$ and the BA with $\sigma_{b}=1 \mathrm{~m}$ and bias $\Delta h_{0}$ modeled as random constant with zero mean and standard deviation $\sigma_{h_{0}}=30 \mathrm{~m}$. The accelerometer sampling rate is $1000 \mathrm{~Hz}$, whereas the BA sampling rate is $100 \mathrm{~Hz}$.

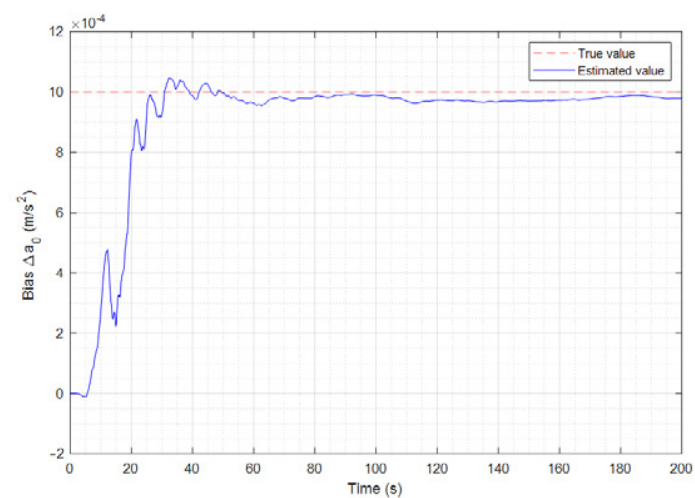

a)
It is considered that, at the initial moment, the aircraft is in a steady-state level flight (altitude $500 \mathrm{~m}$ and speed 80 $\mathrm{m} / \mathrm{s}$ ) heading forward to the glide-slope line. From here, the initial state of Kalman filter is taken as zero for all its elements. The initial value of the covariance matrix of estimation errors is set to (29):

$$
\mathbf{P}_{0}=\left[\begin{array}{ccccc}
10^{2} & 0 & 0 & 0 & 0 \\
0 & 4^{2} & 0 & 0 & 0 \\
0 & 0 & 10^{-6} & 0 & 0 \\
0 & 0 & 0 & 10^{-6} & 0 \\
0 & 0 & 0 & 0 & 10^{2}
\end{array}\right] .
$$

Given flight dynamics equations (15-20) with aerodynamic model (21-23), as well as automatic control system models (25-28) and (31-34), the aircraft motion was simulated in Matlab/Simulink environment.

\subsection{Simulation results without calibration maneuver}

Figures 5 and 6 present the calibration results obtained in a stabilized level flight, i.e., without any maneuver. One can find that the accelerometer bias $\Delta a_{0}$ can be quite accurately calibrated, while the scale factor $\delta k_{a}$ can not be identified.

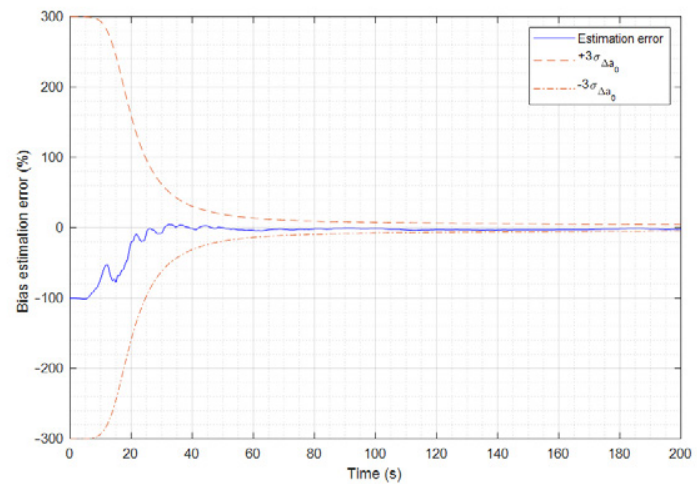

b)

Figure 5. Bias estimation during stabilized level flight: $\mathrm{a}$ - estimated value; $\mathrm{b}$ - estimation error 


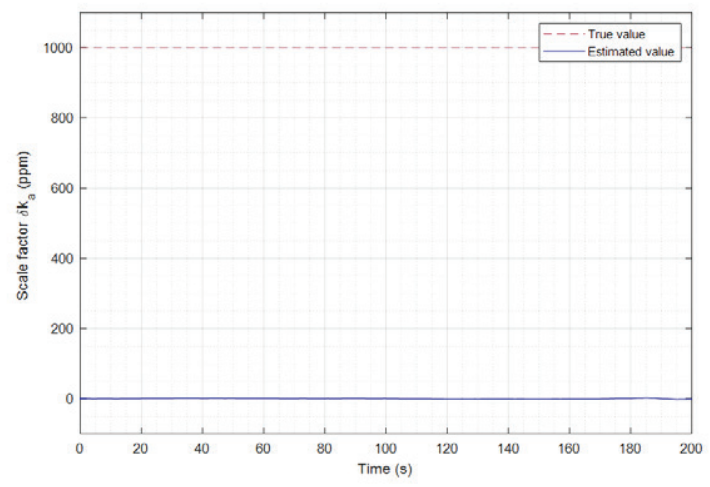

a)

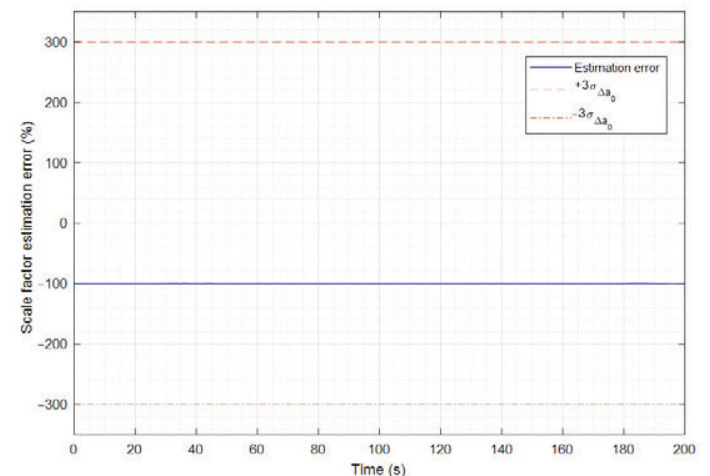

b)

Figure 6. Scale factor estimation during stabilized level flight: $\mathrm{a}$ - estimated value; $\mathrm{b}$ - estimation error

The time history plots in Figure 5 show that the estimation error fairly converges towards zero. After $30 \mathrm{~s}$ the estimated value of bias almost approaches its actual value. At the same time, as shown in Figure 6, the estimated value of scale factor does not change over time and is always close to zero, while its actual value is given as $1000 \mathrm{ppm}$. These simulation results are consistent with the conclusion theoretically made in section 3 by analyzing equation (24). Thus, as expected, any maneuver is required in order to increase the calibration accuracy.

\subsection{Selection of maneuver optimizing vertical accelerometer calibration}

To study the influence of flight maneuver on calibration accuracy, a calibration algorithm was simulated with maneuvers controlled by the altitude/speed hold autopilot (see Figure 4) with various reference altitude changes $\Delta h_{r}=-100 \div 100 \mathrm{~m}$ and command durations $\Delta T_{c m d}=$ $7,8,9,10,11,12,13,14 \mathrm{~s}$.

To examine calibration accuracy, we introduce the relative calibration error calculated from the steady-state values of bias and scale factor, which are estimated by Kalman filter, in the form of:

$$
\delta x(\%)=\frac{\bar{x}-x_{\text {true }}}{x_{\text {true }}} \cdot 100 \%,
$$

where $\bar{x}$ - mean of steady-state estimates of parameter $x$, $x_{\text {true }}-$ true value of $x$.

The simulation results (with flight duration $200 \mathrm{~s}, t_{0}=$ 20 s) are presented in Figures 7 and 8.

As shown in Figure 7, the bias calibration accuracy for any of the planned maneuvers is quite high (the error is not more than $1.1 \%$, the standard deviation of error is less than $1.5 \%)$. This is very important because any desirable maneuver should affect only the scale factor calibration process, but not the bias calibration, which is well performed in a level flight.

Figure 8 presents the results of scale factor calibration. According to them, the command durations $\Delta T_{c m d}$

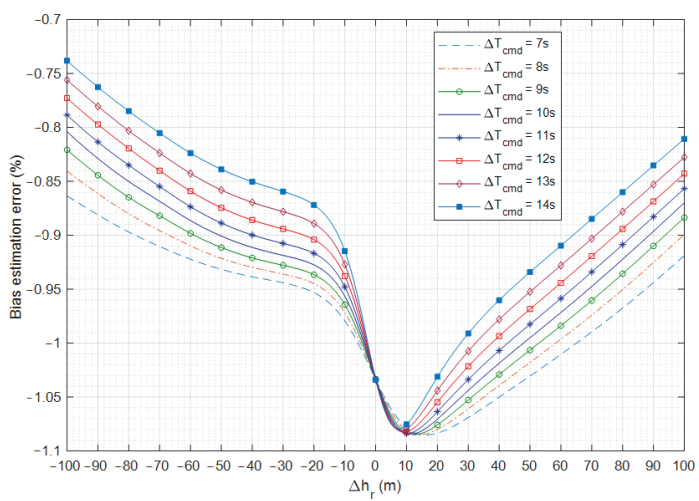

Figure 7. Result of bias calibration with various maneuvers

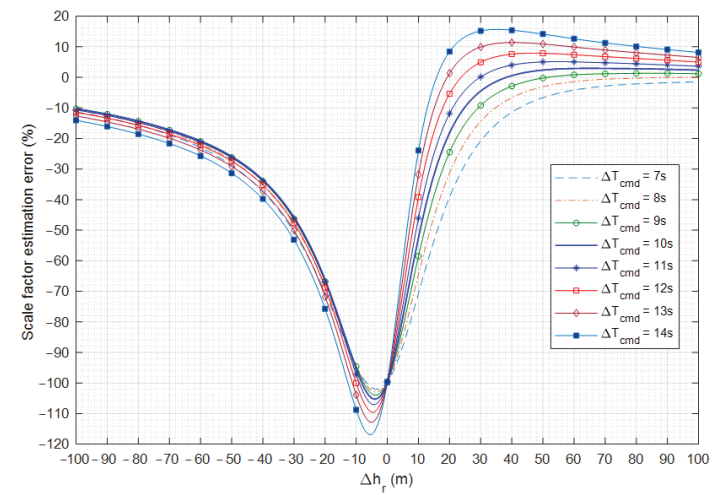

Figure 8. Result of scale factor calibration with various maneuvers

and the corresponding ranges of reference altitude change $\Delta h_{r}$, at which the scale factor calibration error (30) is guaranteed no more than $2 \%$, are shown in Table 1 .

As can be seen from the obtained results, the longer the command duration $\Delta T_{c m d}$ is, the smaller the altitude change $\Delta h_{r}$ is required, and therefore less energy is spent on maneuvering. However, when $\Delta T_{c m d} \geq 11 \mathrm{~s}$, the calibration accuracy is too sensitive to $\Delta h_{r}$ : the scale factor 
Table 1. Required value of reference altitude change

\begin{tabular}{|c|c|c|c|c|c|c|c|c|}
\hline$\Delta T_{c m d}$ & $7 \mathrm{~s}$ & $8 \mathrm{~s}$ & $9 \mathrm{~s}$ & $10 \mathrm{~s}$ & $11 \mathrm{~s}$ & $12 \mathrm{~s}$ & $13 \mathrm{~s}$ & $14 \mathrm{~s}$ \\
\hline$\Delta h_{r}$ & $\geq 90 \mathrm{~m}$ & $\geq 56 \mathrm{~m}$ & $\geq 42 \mathrm{~m}$ & $\geq 32 \mathrm{~m}$ & $26-34 \mathrm{~m}$ & $20-25 \mathrm{~m}$ & $18-21 \mathrm{~m}$ & $14-16 \mathrm{~m}$ \\
\hline
\end{tabular}

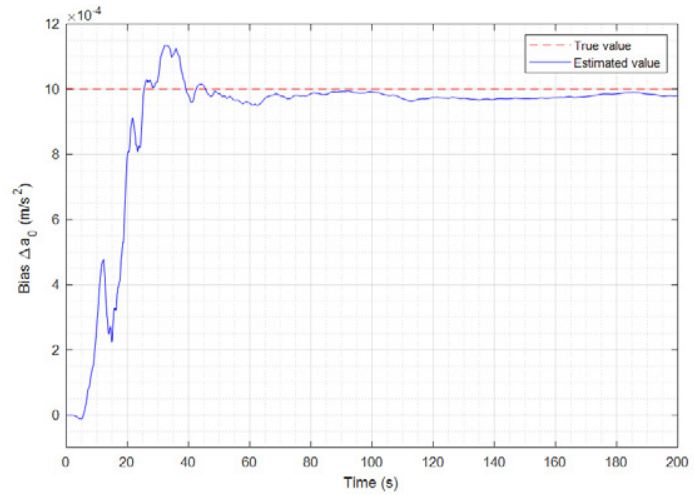

a)

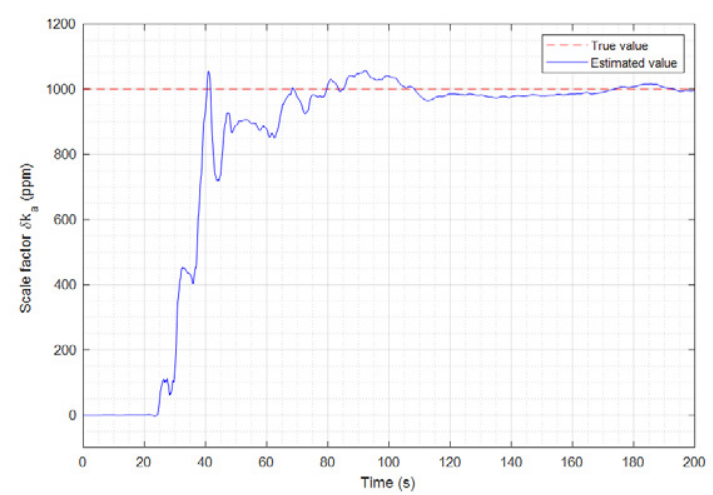

b)

Figure 9. Calibration result with $\Delta T_{c m d}=10 \mathrm{~s}, \Delta h_{r}=38 \mathrm{~m}$ : a - bias; b - scale factor

estimation error curves intersect the zero-error line with large slopes. Thus, to obtain high calibration accuracy, it is advisable to choose a maneuver with $\Delta T_{c m d}=10 \mathrm{~s}$ and $\Delta h_{r}$ no less than $32 \mathrm{~m}$ (the best value of $\Delta h_{r}$ is $38 \mathrm{~m}$, see Figure 8). Figure 9 illustrates the calibration result achieved from one of the appropriate maneuvers.

\subsection{Simulation of aircraft landing with failed BA}

To examine the appropriateness of the proposed calibration algorithm, we simulated the landing (from glideslope capture to flare) of a passenger aircraft introduced in section 2 with BA failure. The aircraft motion is driven by an automatic control system using only feedback signals from a standalone INS. Three simulation scenarios were performed:

1. Scenario 1: calibration was not performed until BA failure. In this case, after BA failure, the initial error model of the vertical accelerometer is used in INS.
2. Scenario 2: before BA failure, the calibration was performed in a normal stabilized level flight, i.e., without any calibration maneuver. In this case, after BA failure, only the estimated value of bias is used in INS to provide additional error compensation.

3. Scenario 2: before BA failure, the calibration was performed with calibration maneuver allowing to estimate not only the bias but also scale factor. After BA failure, the estimated values of both bias and scale factor are used in INS to provide additional error compensation.

The level flight at $500 \mathrm{~m}$ altitude and speed $80 \mathrm{~m} / \mathrm{s}$ before glide-slope capture is stabilized by the altitude/speed hold autopilot shown in Figure 4. For glide-slope capture, an autopilot whose block diagram illustrated in Figure 10 is used.

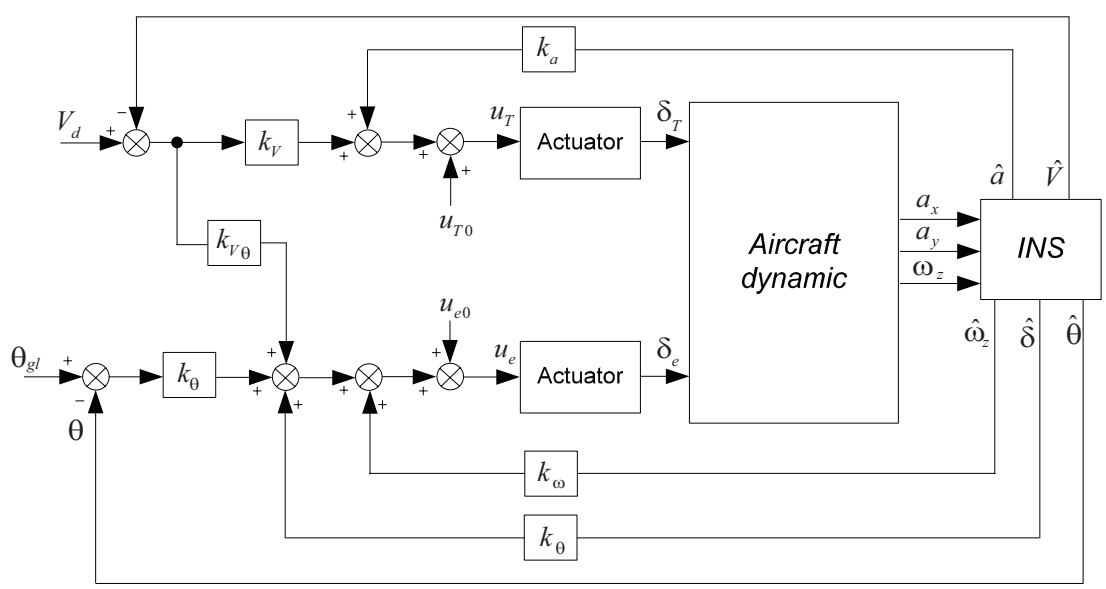

Figure 10. Glide-slope coupler 
Accordingly, control inputs are formed as:

$u_{T}=u_{T 0}+k_{V}\left(V_{d}-\hat{V}\right)+k_{a} \hat{a} ;$

$u_{e}=u_{e 0}+k_{\omega} \hat{\omega}_{z}+k_{\theta}\left(\theta_{g l}-\hat{\theta}\right)+k_{V \theta}\left(V_{d}-\hat{V}\right)+k_{\delta} \hat{\delta}$,

where $u_{T 0}, u_{e 0}$ - program controls corresponding to the trim condition (flight path angle $-3 \mathrm{deg}$, speed $80 \mathrm{~m} / \mathrm{s}$ ); $\theta_{g l}=-3 \mathrm{deg} ; \delta$ - glide path deviation, $\frac{d}{d t} \delta=V \sin \left(\theta-\theta_{g l}\right)$ (Brian \& Frank, 2003); $\hat{V}, \hat{a}, \hat{\omega}_{z}, \hat{\theta}, \hat{\delta}$ - INS based estimate of velocity, acceleration, pitch angle rate, flight path angle and glide path deviation; $k_{V}=9.26, k_{a}=-22.27$, $k_{V \theta}=-0.44, k_{\omega}=1.22, k_{\theta}=-2.12, k_{\delta}=0.08$.

The automatic flare control system is turned on at an altitude of $15 \mathrm{~m}$ and forms the following control inputs (Brian \& Frank, 2003):

$u_{T}=u_{T 0}+\frac{u_{T_{\text {idle }}}-u_{T 0}}{T_{f l}} ;$

$u_{e}=u_{e 0}+k_{1} x_{1}+k_{2} \varepsilon+k_{3}\left(h_{r}-\hat{h}\right)+k_{4} \hat{\omega}_{z}+k_{5} \hat{\theta}+k_{6} h_{r}$,

where $u_{T_{i d l e}}$ - throttle deflection, corresponding to idle mode of an engine; $T_{f l}$ - desired flare time; $h_{r}$ - desire flare trajectory, $\frac{d}{d t} h_{r}=-0.3 h_{r}+r, r=0, h_{r}=15 \mathrm{~m}$ at the start of the flare (Brian \& Frank, 2003); $\hat{\omega}_{z}, \hat{\theta}, \hat{h}-$ INS based estimate of pitch angle rate, flight path angle and altitude; $k_{1}=-918.8, k_{2}=999.89, k_{3}=-76.62, k_{4}=$ $-6.64, k_{5}=-77.28, k_{6}=0.054$.

A block diagram of the presented automatic flare control is shown in Figure 11.

The simulation ends when the aircraft touches the runway, either the based INS estimate of altitude is equal to zero. The simulation results are shown in Figure 12 and Table 2. For convenience of analysis, landing trajectories are presented only in the last seconds.

As can be seen from Figure 12, additional INS error compensation using the calibration results (lines $h_{1}$ and $h_{2}$ ) is needed to prevent a crash-landing (line $h_{3}$ ). From the data in Table 2, we find that in scenario 1 the vertical speed and the flightpath angle at touchdown are too large and therefore cause the aircraft to fall to the ground. It

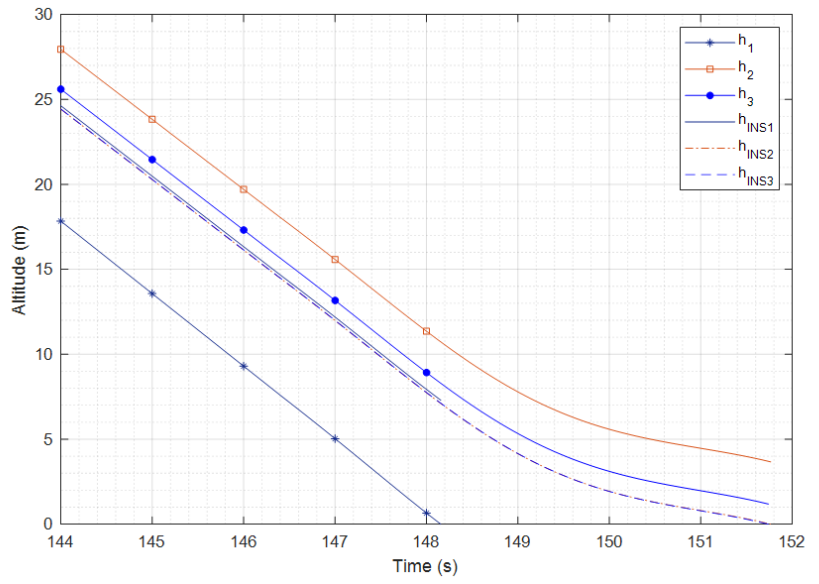

Figure 12. Three landing simulation scenarios $\left(h_{i}, h_{\mathrm{INS} i}\right.$, $i=1,2,3$ - altitude and its based INS estimate by scenario)

Table 2. Final state of the aircraft

\begin{tabular}{|c|c|c|c|c|}
\hline Scenario & $h(\mathrm{~m})$ & $V_{y}(\mathrm{~m} / \mathrm{s})$ & $\omega_{z}(\mathrm{deg} / \mathrm{s})$ & $\theta(\mathrm{deg})$ \\
\hline 1 & 0 & -4.1 & 1.2 & -2.8 \\
\hline 2 & 3.67 & -1.317 & -2.289 & -0.952 \\
\hline 3 & 1.18 & -1.318 & -2.293 & -0.950 \\
\hline
\end{tabular}

can be explained, focusing to lines $h_{1}$ and $h_{\mathrm{INS} 1}$ : when the aircraft is at about $5 \mathrm{~m}$ above the ground, due to inaccurate altitude information (estimation error is about $7 \mathrm{~m}$ ) from INS, the automatic flare control system still forces the aircraft to track the straight-line path of desired flare trajectory (which is very close to line $h_{\mathrm{INS3}}$ ). As a result, the aircraft continues to descend at high vertical speed and hits the ground.

In contrast, when the error model of vertical accelerometer is adjusted by the calibration results (scenarios 2 and 3), a soft landing can be expected based on behaviors of aircraft trajectories in the last moment (see lines $h_{2}$ and $h_{3}$ ). Indeed, Table 2 shows that, when the aircraft is almost close to the ground, its vertical speed is about $-1.3 \mathrm{~m} / \mathrm{s}$, the flightpath angle is less than 1 degree and the aircraft is

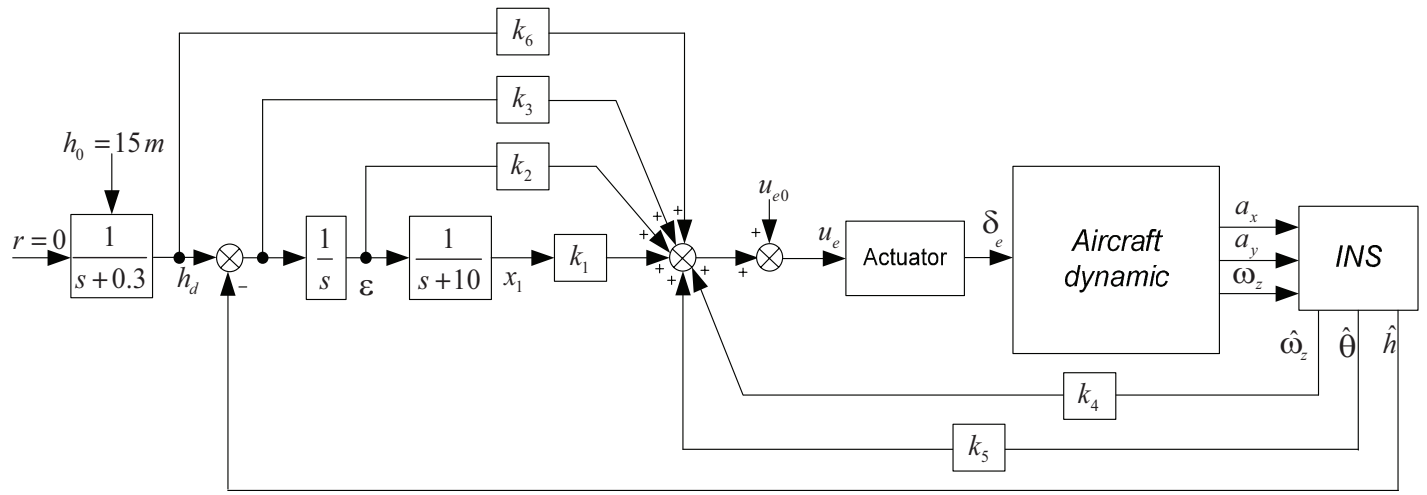

Figure 11. Automatic flare control 
pitching up ( $\left.\omega_{z} \approx-2.3 \mathrm{deg} / \mathrm{s}\right)$. This is a significant performance improvement in comparison to scenario 1 .

Now let's see the impact of the calibrated bias and scale factor of vertical accelerometer on altitude estimation error. To simplify the analysis, altitude estimation errors in three scenarios were taken at the moment of crash-landing occurring in scenario 1 (i.e., at $\mathrm{t}=148.2 \mathrm{~s}$ ): $\Delta h_{1} \approx 7 \mathrm{~m}$, $\Delta h_{2} \approx 3.5 \mathrm{~m}, \Delta h_{3} \approx 1 \mathrm{~m}$. In scenario 1 the altitude estimation error is largest because no error compensation is made. In scenario 2 the bias compensation is provided, reducing the altitude estimation error from $7 \mathrm{~m}$ to $3.5 \mathrm{~m}$. The effectiveness of the proposed calibration algorithm follows the comparison between scenarios 2 and 3: additional scale factor compensation in scenario 3 reduces the altitude estimation error by another more $2.5 \mathrm{~m}$ (from 3.5 $\mathrm{m}$ to $1 \mathrm{~m}$ ). Thus, if the calibration is performed with a designed calibration maneuver, it is possible to significantly reduce the altitude estimation error, which means that a more successful landing can be achieved.

\section{Conclusions}

An approach to additional (in-flight) vertical accelerometer calibration, based on refining its characteristics through a specific aircraft maneuver, was investigated. Validity and performance of the proposed algorithm were assessed by simulations of flight dynamics and aerodynamic models of a passenger aircraft, and baro-inertial integration using Kalman filter and its associated models. Applying designed calibration maneuver before capturing the glide-slope, simulation results show acceptable landing accuracy and safety level with only feedback signal from standalone INS (assuming a BA failure). Moreover, the proposed scheme for the measurement signal generation used in Kalman filter confirms that the influence of BA bias on the calibration accuracy can be eliminated, allowing a relevant calibration of the vertical accelerometer without the need to estimate the BA bias.

In this paper, the time constant $\tau$ of BA is assumed known exactly, using equation (4). However, in practice, it is required to adjust $\tau$ for obtaining acceptably accurate estimate of altitude changes. This requires a more complicated integrated system for calibrating $\tau$ using GNSS and optical navigation system in further work. A nonlinear estimation with extended or unscented Kalman filter could also be an option for the next development. Investigation on the Pitot port blockage will also be considered in the future work to better cover the flight safety, in particular for incidents from air data computer failures.

\section{Author contributions}

Man Nguyen and Vyacheslav Kostiukov conceived of the proposed idea, developed the mathematical models and algorithms. Man Nguyen and Cap Tran performed the simulation, data collection, and analysis. Vyacheslav Kostiukov and Cap Tran wrote the first draft of the manuscript. Man Nguyen revised the manuscript.

\section{Disclosure statement}

We have not any competing financial, professional or personal interests from other parties.

\section{References}

AAIU. (2016). Synoptic report. Serious incident Boeing 757-224, N41140 80 NM Southwest of Dublin, Ireland, 20 October 2013. Air Accident Investigation Unit, Ireland.

Ausman, J. S. (1991). A Kalman filter mechanization for the Baro-inertial vertical channel. Proceedings of the 47th Annual Meeting of the Institute of Navigation, 1, 153-159.

Babich, O. A. (1991). Obrabotka informatsii v navigatsionnykh kompleksakh. Mashinostroenie (in Russian).

BEA. (2012). Final report on the accident on $1^{\text {st }}$ June 2009 to the Airbus A330-203 registered F-GZCP operated by Air France flight AF 447 Rio de Janeiro - Paris. French Civil Aviation Safety Investigation Authority.

Bevermeier, M., Walter, O., Peschke, S., \& Haeb-Umbach, R. (2010). Barometric height estimation combined with mapmatching in a loosely-coupled Kalman-filter. 7th workshop on positioning, Navigation and Communication (pp. 128-134). Dresden, Germany. https://doi.org/10.1109/WPNC.2010.5650745

Brian, L. S., \& Frank L. L. (2003). Aircraft control and simulation (2nd ed.). John Wiley \& Sons.

Farrell, J., \& Barth, M. (1998). The global positioning system and inertial navigation. McGraw-Hill.

FAA. (2016). Pilot's handbook of aeronautical knowledge. United States Department of Transportation, Federal Aviation Administration.

Gray, R. A., \& Maybeck, P. S. (1995). An integrated GPS/INS/ BARO and radar altimeter system for aircraft precision approach landings. Proceedings of IEEE 1995 National Aerospace and Electronics Conference (pp. 161-168). Dayton, OH, USA.

Groves, P. D. (2013). Principles of GNSS, inertial, and multisensor integrated navigation systems (2nd ed.). Artech House.

Groves, P. D. (2015). Navigation using inertial sensors [Tutorial]. IEEE Aerospace \& Electronics Systems Magazine, 30(2), 42-69. https://doi.org/10.1109/MAES.2014.130191

Jafar, K., Hossein, N., \& Sadra, R. (2018). Design and Implementation of GA Filter Algorithm for Baro-inertial altitude error compensation. 18th IIE International Conference on Latest Trends in Engineering and Technology (pp. 78-80). Istanbul, Turkey.

Jeb, B. (2019). Pitot-static system failures. Aviation Safety. https:// www.aviationsafetymagazine.com/features/pitot-static-system-failures/

Kim, J. H., \& Sukkarieh, S. (2003). A baro-altimeter augmented INS/GPS navigation system for an uninhabited aerial vehicle. Paper presented at the 6th International Symposium on satellite navigation technology including mobile positioning \& location services. Melbourne, Australia.

KNKT. (2018). Aircraft accident investigation report. Komite Nasional Keselamatan Transportasi, Republic of Indonesia.

Lawford, J. A., \& Nippress, K.R. (1983). Calibration of air-data system and flow direction sensors. AGARD-AG-300. Volume 1.

Luiz, R. M. (2013). The dramatic effect of Pitot-Static system blockages and failures. http://www.luizmonteiro.com/DocumentsPDF/The_Dramatic_Effects_of_Pitot_Static_Blockages.pdf

Mohinder, S. G., Lawrence, R. W., \& Angus, P. A. (2007). Global positioning systems, inertial navigation, and integration (2nd ed.). Wiley. 
O’Donnel, C. F. (1964). Inertial navigation, analysis and design. McGraw-Hill.

Siouris, G. M. (1993). Aerospace avionics system: a modern synthesis. Academic Press, Inc.

Schmidt, G. T. (2010). INS/GPS technology trends. NATO RTO Lecture Series, RTO-EN-SET. Massachusetts, USA.

Sobolev, V. I. (1994). Syntes kalmanovskikh fil'trov: Ucheb. posobie dlia prakticheskykh zaniatiu. Izd-vo MAI (in Russian).
Sokolovi, V. S., Diki, G., \& Stan, R. (2014). Adaptive error damping in the vertical channel of the INS/GPS/Baro-altimeter integrated navigation system. Scientific Technical Review, 4(3), 141-150.

Zaporozhets, A. V., \& Kostiukov, V. M (1992). Proektirovanie system otobrazheniia informatsii: Ucheb. posobie dlia priborostroitel'nykh spetsial'nostě vuzov. Mashinostroenie (in Russian). 